\title{
ACUTE OSTEOMYELITIS IN CHILDREN
}

\author{
R. A. B. Mollan and J. Piggot, Belfast, Northern Ireland \\ From the Royal Belfast Hospital for Sick Children, the Ulster Hospital, Dundonald, and the \\ Withers Orthopaedic Centre, Belfast
}

\begin{abstract}
A number of problems in the treatment of acute osteomyelitis have remained unresolved in recent years. The clinical experience of ninety-three patients with proven acute haematogenous osteomyelitis is presented to help resolve these problems. Analysis of the clinical features, the operative, bacteriological and haematological findings is made and discussed in detail. Eighteen patients had continuing bone infection and recommendations are made as to how diagnosis and management might have been improved. Surgery is considered to be an essential part of the diagnostic and therapeutic management of this disease. A combination of cloxacillin and fusidic acid is recommended as the antibiotic treatment.
\end{abstract}

In recent years a number of problems have been defined in the treatment of acute osteomyelitis. The place of operation continues under review and, although its place in the treatment of the disease was established by Harris (1962) and by Trueta and Morgan (1954), it was postulated later that this was not proven to be essential. A leading article (British Medical Journal 1972) considered that surgical drainage was essential only in the presence of clear clinical evidence of a subperiosteal abscess and it was alleged that routine exploration might even result in harm. This view was hotly disputed by Mullick (1972) and by Merryweather (1972). The choice of antibiotics is of paramount importance because of the emergence of resistant bacteria, the great variety of antibiotics available and interactions between drugs. Constant surveillance is therefore needed. Rest and splintage are essential aspects of treatment but the place of operation and the best choice of antibiotic are aspects which have yet to be resolved.

This paper presents the clinical experience of ninetythree patients with proven acute osteomyelitis as a contribution to further consideration of the effective treatment of this disease.

\section{MATERIAL AND METHODS}

All patients with primary bone infection who presented to two Belfast hospitals* from 1960 to 1974 were reviewed. Patients in whom the diagnosis was made on clinical grounds only, or in whom there was insufficient information recorded, were excluded from the survey. All patients with continuing bone infection were included. One hundred and ninety patients were surveyed-an average presentation of one every month. Ninety-three patients fulfilled the criteria of diagnosis defined by Blockey and Watson (1970). A variety of antibiotic regimes were used and not all the patients had surgical exploration. Patients were under the care of either general, paediatric or orthopaedic surgeons. The average follow-up was two years six months, the shortest being three months and the longest twelve years.

\section{RESULTS}

Of the ninety-three patients in the survey, fifty-two were boys and forty-one girls. There was not the expected male preponderance. The ages ranged from two weeks to sixteen years (Fig. 1). The sites of infection and their

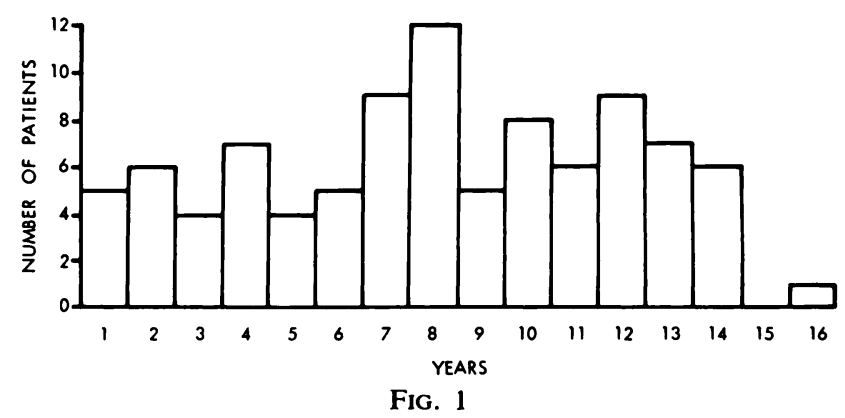

Age distribution of patients with acute osteomyelitis.

incidence at each site is shown diagrammatically in Figure 2. Four patients had more than one site of infection. There was no preference for either side of the body. There was no correlation between site and length of time before diagnosis. In only twenty-nine per cent of cases could a history of injury be considered relevant.

On admission all but four patients had pyrexia (Figs. 3 and 4). Most patients who responded to treatment showed a steady decrease in temperature to normal limits within five days of admission. Eighteen patients progressed to chronic osteomyelitis; of these only four had a normal temperature at five days; the others had an intermittent pyrexia sometimes taking weeks to settle.

\footnotetext{
* Royal Belfast Hospital for Sick Children and the Ulster Hospital, Dundonald.
} 
Sixty-eight patients were toxaemic on admission, and of the forty-nine patients who had blood cultures taken, twenty-two had bacteraemia. There were two patients with septicaemia. In this survey there were no clinical conditions which seemed to predispose to acute osteomyelitis.

Not all of the patients presented with cardinal signs of bone infection. Loss of function of a limb was an important sign in young children and babies. The severity of the clinical signs did not always reflect the severity of bone infection found at surgical exploration, and subperiosteal pus was found in the absence of clinical signs of abscess formation. The finding of pus at operation was not related to delay in diagnosis. Twenty-two patients were seen within forty-eight hours of the onset of symptoms and twenty-one of these were found to have pus at operation. Sixty-six patients were explored surgically and fifty-nine were found to have pus.

Twenty-seven patients were not submitted to operation and were treated with antibiotics and splintage. In these patients the clinical signs were very slight and responded rapidly to treatment, and none progressed to chronic osteomyelitis.

The erythrocyte sedimentation rate was elevated on admission in fifty-six patients out of the fifty-nine for whom records were available; in two patients the level was above 100 millimetres in the first hour. Elevation of the sedimentation rate after six weeks indicated continuing bone infection. It was noted that in the patients who proceeded to chronic osteomyelitis there was a steady drop in the level for five to seven weeks after admission, but later the level began to rise and remain elevated. The white cell count was elevated on admission in forty-two of seventy-three patients recorded; all had returned to normal limits within two weeks. Patients who progressed to chronic osteomyelitis did not have an elevated white cell count. The average haemoglobin level on admission was 12 grams per 100 millilitres of blood.

Organisms were isolated from blood culture and pus in sixty-two patients. The blood of forty-nine patients was cultured on admission, organisms being isolated from twenty-two. In fifty-four out of fifty-nine patients organisms were isolated from pus. In three patients in whom pus was not found at operation organisms were isolated from oedema fluid. The staphylococcus aureus was isolated in ninety-three per cent of cultures and seventy-four per cent of these were resistant to benzylpenicillin (Tables I and II).

Eighteen patients progressed to chronic osteomyelitis; these were analysed in detail (Table III). Eleven patients were treated with antibiotics in the early stages which proved to be inadequate treatment, because of low dosage, wrong route of administration or organism insensitivity. In ten patients the diagnosis was reached after a long delay. Six patients either had no operation or an inadequate procedure. Four of the eighteen patients were found to have no obvious reason why they

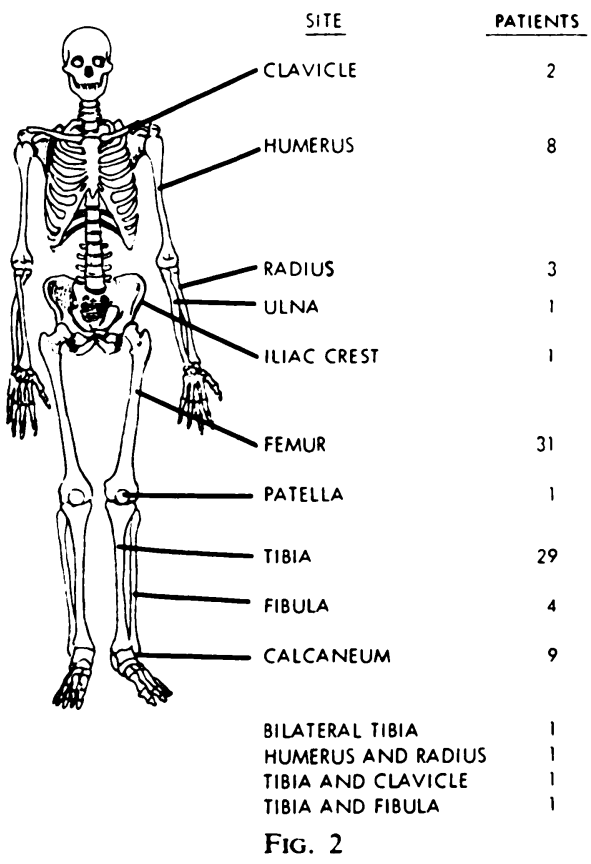

Incidence of bone involvement at various sites.

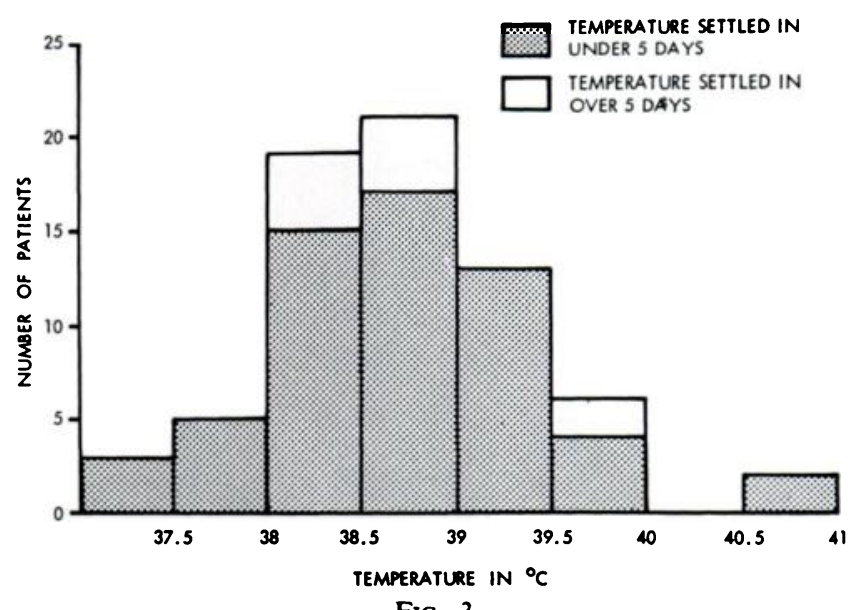

Fig. 3

Histogram of temperature on admission of those patients who responded to primary treatment. (Two patients had no temperature recorded.)

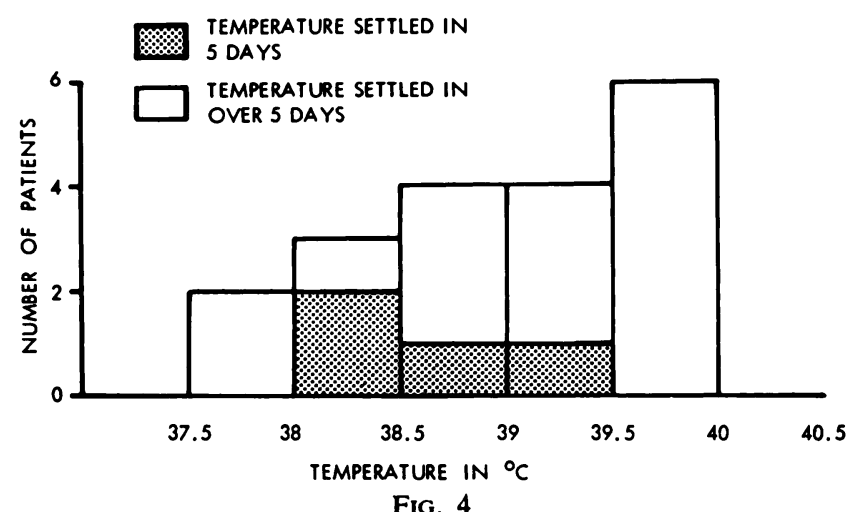

Histogram of temperature on admission of those patients who had continuing bone infection. 
TABLE I

Organisms Isolated from Pus and Blood Culture

\begin{tabular}{|ll|c|}
\hline \multicolumn{1}{|c|}{ Organism } & Number isolated \\
\hline Staphylococcus aureus . & $\cdot$ & 58 \\
Staphylococcus albus . &. & 2 \\
Streptococcus pyogenes & $\cdot$ & 1 \\
Streptococcus viridans . & $\cdot$ & 1 \\
\hline
\end{tabular}

TABLE II

Resistance to ANtibiotics

\begin{tabular}{|c|c|}
\hline Drug & Number of cases \\
\hline Benzylpenicillin & 15 \\
\hline Benzylpenicillin and ampicillin . & 18 \\
\hline $\begin{array}{l}\text { Benzylpenicillin, ampicillin and } \\
\text { fusidic acid }\end{array}$ & 2 \\
\hline $\begin{array}{l}\text { Benzylpenicillin, ampicillin and } \\
\text { tetracyline. }\end{array}$ & 8 \\
\hline
\end{tabular}

TABLE III

ANALYSIS OF FaILURES

\begin{tabular}{|c|c|}
\hline Reason for failure & Number of cases \\
\hline Inadequate antibiotics alone & 7 \\
\hline $\begin{array}{l}\text { Inadequate antibiotics plus in- } \\
\text { adequate surgery }\end{array}$ & 2 \\
\hline $\begin{array}{l}\text { Inadequate antibiotics plus delay } \\
\text { in diagnosis }\end{array}$ & 2 \\
\hline Inadequate surgery & 3 \\
\hline No obvious reason. & 4 \\
\hline
\end{tabular}

progressed to chronicity. All patients who developed chronic bone infection showed this within three months of discharge from hospital and while still under review. In fourteen patients the bone infection continued from the start, and of the other four one presented at one month, two at two months and one at three months after discharge.

\section{ILLUSTRATIVE CASES}

Case 1-A baby girl aged two weeks was admitted with irritability and a pyrexia of $\mathbf{3 9}$ degrees Celsius. A diagnosis of otitis media and a respiratory infection was made. However, the abdomen was swollen and she was admitted to a surgical ward with a provisional diagnosis of intestinal obstruction. She had been a difficult forceps delivery and had haemolytic disease of the newborn. A blood transfusion was given to correct a severe anaemia and ampicillin was given to treat her chest infection. Her abdomen was observed. She did not settle and she was later transferred to a medical ward where the diagnosis of staphylococcal septicaemia was made. Blood culture grew a staphylococcus aureus resistant to benzylpenicillin and ampicillin. She remained ill with a diagnosis of staphylococcal pneumonia. Three weeks after admission a routine chest radiograph revealed, by accident, a gross osteomyelitis of the left humerus (Figs. 5 and 6). The shoulder was very swollen and the arm was immobile. Aspiration revealed thick pus from which staphylococci were grown. The organisms were resistant to penicillin and ampicillin. Surgical drainage, delayed a further three days because of a presumptive diagnosis of septic arthritis, revealed a huge subdeltoid abscess. Fusidic acid and cloxacillin were then started and the arm was splinted for six weeks. The baby improved dramatically. Follow-up at six years showed 2 inches of shortening of the left arm, full movements and, radiologically, an abnormal humeral head (Fig. 7). At no time had she evidence of continuing bone infection.

Comment-This case illustrates the difficulty of diagnosis in the neonate and the consequences of delay.

Case 2-A boy aged four years was admitted with vomiting, constipation and a tender abdomen. His temperature was 40.2 degrees Celsius. A provisional diagnosis of acute appendicitis was made. Laparotomy revealed a normal appendix but gross mesenteric adenitis. Two days later he was still toxic and it was noticed that he had a red tender area at the upper end of his left tibia (Fig. 8). Exploration was carried out and a tense subperiosteal abscess was drained. Culture of the pus produced staphylococcus aureus resistant to penicillin and ampicillin. He was given tetracycline and streptomycin, then changed to cloxacillin and fusidic acid. Later the next day he complained of pain in his right shoulder (Fig. 9). His right clavicle was tender and inflamed but it was considered unlikely to be osteomyelitis. Finally, three days later it was explored and a subperiosteal abscess drained. Continuing bone infection was present at three months in both sites but by nine months both had settled. Follow-up at five years showed no recurrence or disability.

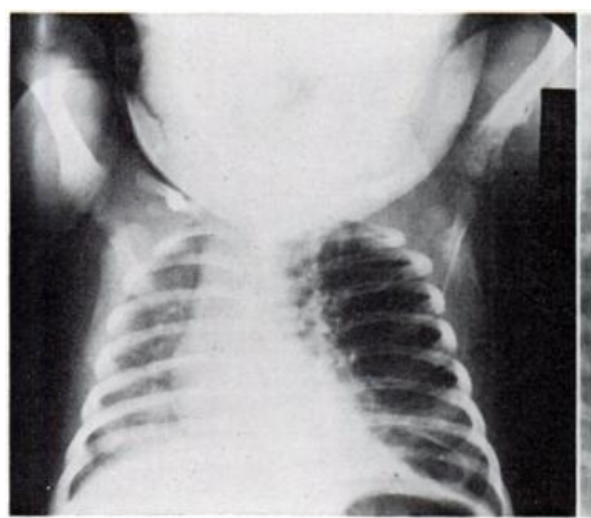

Fig. 5

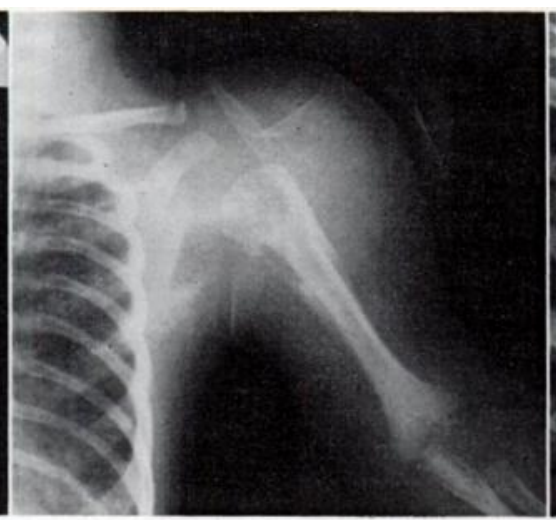

FIG. 6

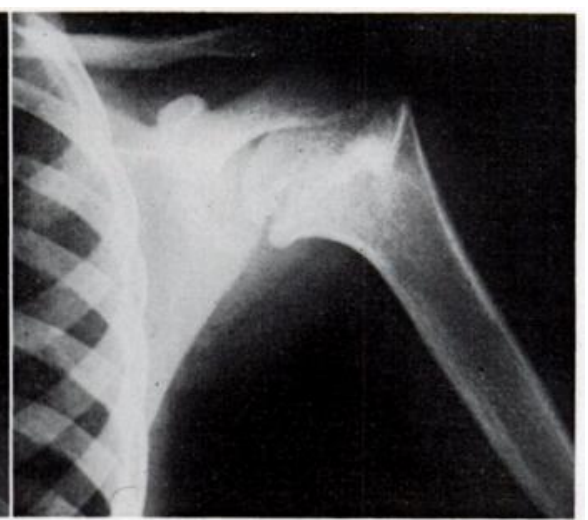

FiG. 7

Case 1. Figure 5-The chest radiograph which revealed the osteomyelitis of the left humerus. Figure 6-Radiograph of the left humerus showing much soft-tissue swelling caused by a subdeltoid abscess. Figure 7-The radiographic appearance of the left humerus six years later. 
Comment-This case illustrates the difficulty in diagnosis in the presence of vomiting and toxaemia. It emphasises the need always to be aware of a double lesion and to differentiate the diagnosis from acute infantile rheumatism.

Case 3-A girl aged two years was admitted in status epilepticus. She had had a septic laceration on her left leg some days previously, which had been followed by fever. She had also had frequency of micturition before admission which was treated with ampicillin at home. On admission her temperature was 41.4 degrees Celsius. The convulsions settled with paraldehyde and phenobarbitone. A diagnosis of meningitis was made, but lumbar puncture was negative. While semiconscious she was noticed to react to pressure on the right thigh; orthopaedic advice was sought. Immediate exploration of the femur was done and bloodstained oedema fluid,

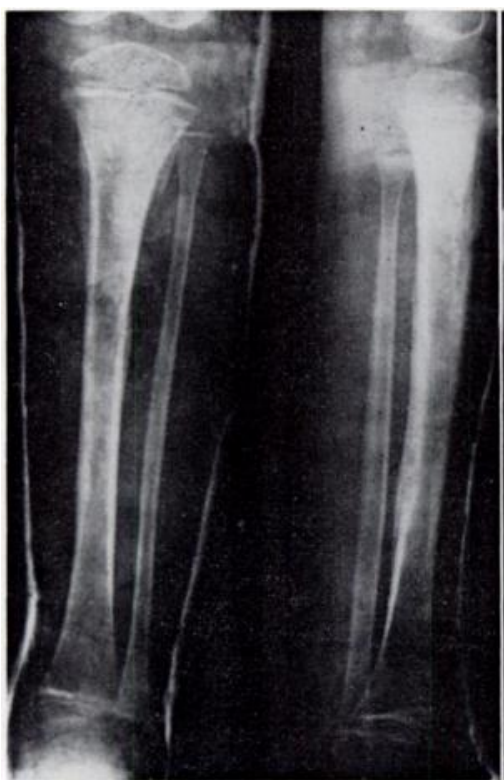

FIG. 8

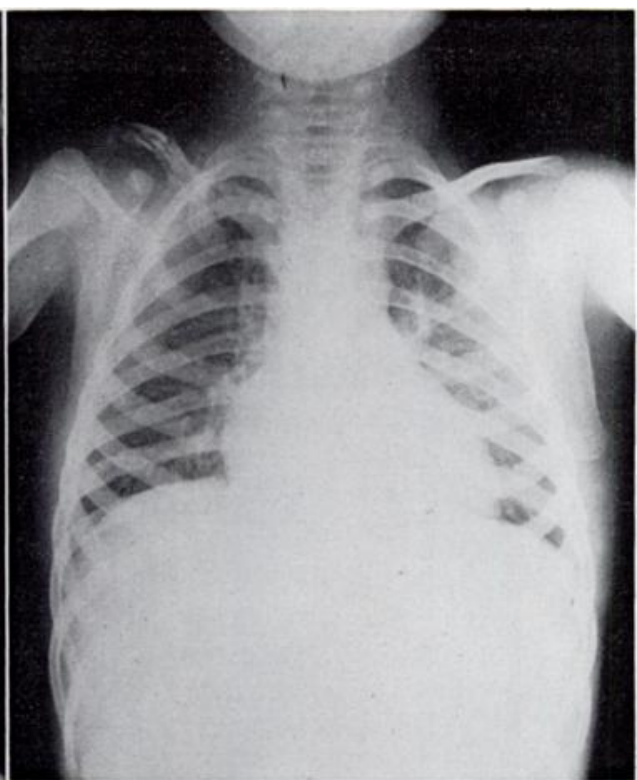

Fig. 9

Case 2. Figure 8-Radiograph of the left tibia and fibula after surgical drainage. Figure 9Radiograph of chest showing osteomyelitis of the right clavicle.

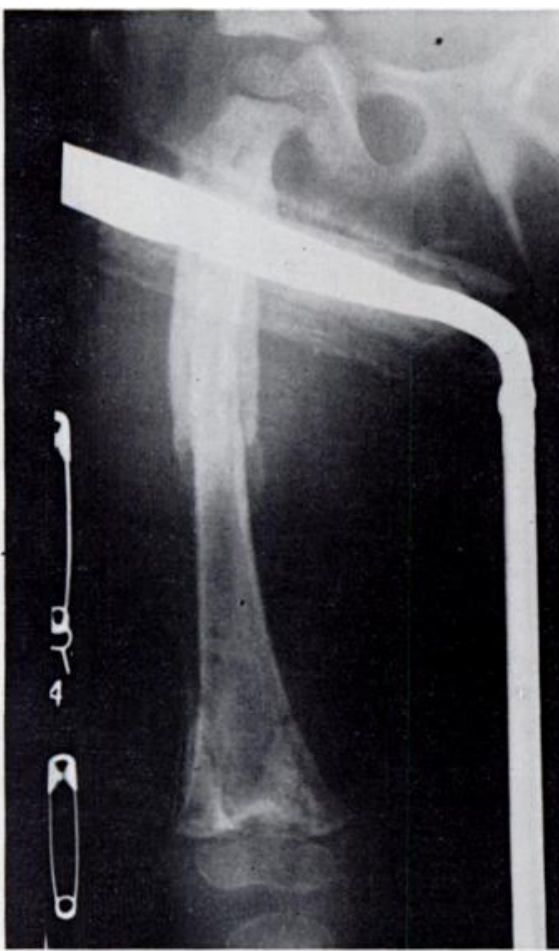

FIG. 10

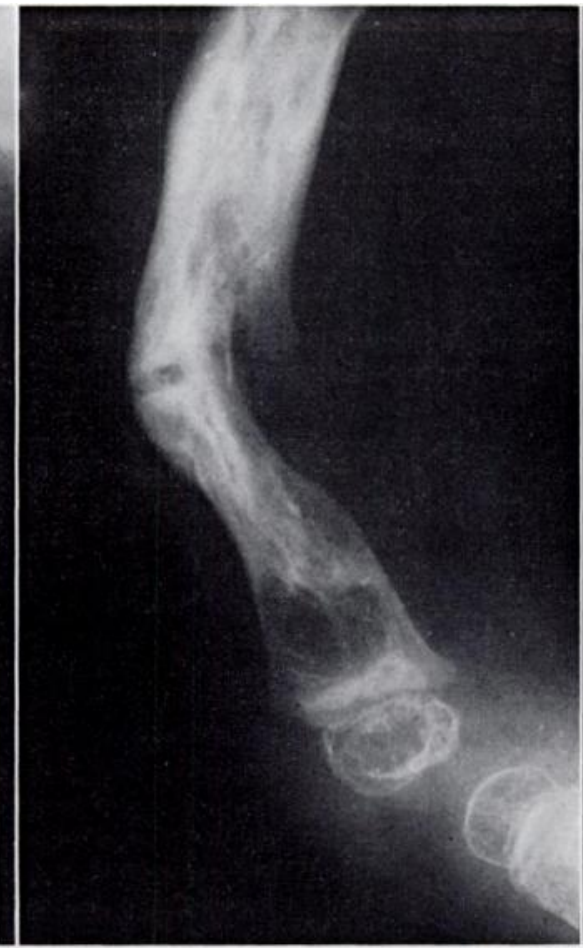

FIG. 11

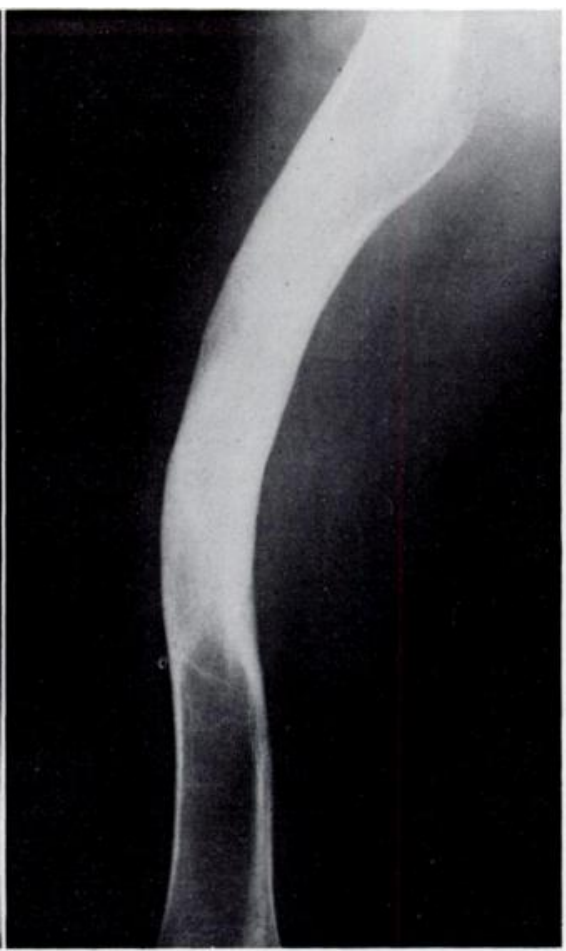

FIG. 12

Case 3. Figure 10-Radiograph of severe chronic osteomyelitis of the right femur with pathological fractures. Figure 11-Radiograph three years after onset. Figure 12-Radiograph eight years after onset. 
which grew a sensitive staphylococcus aureus on culture, welled from the incised periosteum. Fusidic acid and cloxacillin were given. She improved quickly. However, three weeks later while still in hospital, the wound discharged pus, and the radiograph showed severe osteomyelitis and a pathological fracture (Fig. 10). She has had numerous admissions and operations for chronic osteomyelitis and still has a discharging sinus eight years later (Figs. 11 and 12).

Comment-This case illustrates that early exploration led to an early diagnosis in a difficult presentation. However despite this and with adequate antibiotic cover and splintage the good initial response progressed to severe osteomyelitis and its concomitant complications.

Case 4-A girl aged eight years was admitted from a peripheral hospital in a critical condition. For some days she had been ill with an unexplained pyrexia. On admission she was diagnosed as having an acute osteomyelitis of the right ulna with septicaemia and an endocarditis. The haemoglobin was 30 per cent, the white cell count was 2,500 cells per cubic millimetre and the erythrocyte sedimentation rate was 83 millimetres in the first hour. She was given parenteral cloxacillin, a blood transfusion and was taken to theatre where the ulna was explored and pus drained. Her condition had settled within forty-eight hours. A staphylococcus aureus was cultured and at four days the haemoglobin was 80 per cent, white cells were 7,400 per cubic millimetre and the erythrocyte sedimentation rate was $\mathbf{4 2}$ millimetres in the hour. Four weeks later her temperature again became elevated and acute tonsillitis was diagnosed. The following day she developed an acute abdomen and later that night an acutely inflamed appendix was removed. Later, when her stitches were taken out and cloxacillin discontinued her temperature suddenly became elevated and the ulna became inflamed. Antibiotics were again given, the ulna settled, and six weeks later the antibiotics were stopped. Within three days her temperature was 39 degrees Celsius. Examination revealed generalised lymphadenopathy, a palpable spleen and a significantly enlarged liver. The white cells showed 9 per cent abnormal lymphocytes and acute leukaemia was diagnosed after a marrow puncture. The child was treated medically but died soon afterwards with pyaemic abscesses in the liver and spleen.

Comment-Osteomyelitis may be the result of decreased body resistance, and this case illustrates the point that if after a course of adequate treatment antibiotics are discontinued, and the condition recurs, some other underlying pathology must be carefully excluded.

\section{DISCUSSION}

There are many factors which favour early surgical exploration. The recognised surgical treatment of any abscess is drainage to lessen the risks of toxaemia, bacteraemia and septicaemia. Pus was present in 59 out of 66 patients explored and no matter how quickly exploration was performed after the onset of pain, pus was still found. This would disagree with Craven, Pugsley and Blowers (1970) that 95 per cent of patients seen within three days do not need surgical exploration. Here clinical judgement must refute a rigid adherence to a time schedule. We found it clinically impossible to be sure whether a subperiosteal abscess was present or not. We do not think that needle aspiration as recommended by
Weissberg, Smith and Smith (1974) or Bulmer (1967) is adequate. The presence of pus under the periosteum must cause periosteal stripping and endanger the blood supply to bone and it is logical to assume that endangered bone and bacteria in the same area would predispose to chronic infection. In other sites in the body antibiotic treatment of an abscess without drainage only leads to chronic infection or a sterile abscess. The presence of pus in so many cases also gives the surgeon the opportunity to establish the diagnosis, to culture the pus, identify the organism responsible and to ascertain its antibiotic sensitivity. Drilling of the metaphysis has not resulted in harm but delay may damage an epiphysis, halting further growth. We did not find that exploration was carried out in the wrong place and we disagree with Blockey and Watson (1970) that a scar is too high a price to pay for certain diagnosis, bacteriological examination and a good chance of cure. Surgeons dealing with these cases in the primary stages must not be misguided by the leading article in the British Medical Journal (1972) and by the plea by McAllister (1974) for a conservative surgeon. Routine exploration, drainage and culture of pus should be part of the correct management of acute osteomyelitis. Mild clinical types respond to antibiotics alone and represent either good host resistance or poor bacterial aggressiveness. Surgery must aid host resistance in all but the mildest forms of the disease. We should return to the advice of Harris (1962) that operation should not be unduly delayed. Surgery is an essential therapeutic and diagnostic procedure, to be carried out early and as a routine in all but the mildest of cases.

Seventy-four per cent of organisms were resistant to benzylpenicillin. The incidence varies in reported series but it is now agreed that the first choice is a B-lactamaseresistant drug (Lancet 1975). All organisms cultured were sensitive to cloxacillin and as this antibiotic meets the requirements (Green 1967) we now use this as our first choice. Fears of resistance (Blockey and McAllister 1972) have not yet been realised. Fusidic acid has been recommended in combination with other antibiotics as it prevents emergence of resistant strains (Jensen and Lassen 1969). These antibiotics given together as first line treatment, eliminate guesswork from antibiotic cover. We found that adequate treatment did reveal itself in a gradual decrease in temperature and we disagree with Nade (1974) that the temperature in staphylococcal infections takes more than seven days to settle. A good response was seen in our series after adequate surgery. The mode of antibiotic therapy must be dictated by the clinical conditions, with intravenous fusidic acid being a very valuable route in the severe case. Cloxacillin and fusidic acid make a logical choice of antibiotic therapy, and we now use these antibiotics in combination for an average time of six weeks.

Splintage is an essential adjunct to treatment because it is both therapeutic and analgesic, and it should be continued until pain is relieved and the clinical signs of 
activity have gone. Also when there is continuing bone infection splintage is essential to prevent a pathological fracture. The finding of a low haemoglobin on admission and the possibility that it would continue to be so in an infective condition indicates a need for iron and vitamin supplements. Transfusion of whole blood should be considered in all moderate to severe cases.

We found that continuing bone infection had revealed itself within three months of discharge from inpatient care. Craven et al. (1970) believed that infection might recur after years but even in our worst cases, once infection had been controlled, years of follow-up did not reveal any recurrence. Long-term follow-up is essential however if there is any question of abnormal growth of bone. Continuing bone infection revealed itself in a chronic discharge of pus, specific activity on radiographs and an elevated erythrocyte sedimentation rate for more than seven weeks. Analysis of the failures showed that improvement in our practice could be made in a number of ways. The diagnosis of acute osteomyelitis must be considered in all atypical cases of a pyrexial illness of unknown origin and of recent onset. It is essential that, in such an unexplained pyrexia in a child, acute osteomyelitis be considered and excluded by an orthopaedic surgeon. Careful palpation of bone will reveal a site of tenderness in an irritable or functionless limb, especially in the younger age groups. Admission followed by splintage and constant careful review will be rewarded by early diagnosis. Once the diagnosis has been made antibiotics should be given parenterally; we now give cloxacillin and fusidic acid in combination routinely, followed by immediate surgical exploration and decompression in all but the mildest cases. If pus is not found at operation culture of the tissue fluid will very often grow staphylococci. Rest and antibiotics are continued for six weeks as a routine. The erythrocyte sedimentation rate and radiographs are used to monitor progress.

Acute osteomyelitis treated in the correct manner may be just a short episode in the life of a child. If badly managed it is life threatening at worst and, at best, a debilitating, crippling illness. Clearly an orthopaedic surgeon, who often sees the results of disaster, is the best person to advise and treat acute osteomyelitis from the earliest suspicion of this diagnosis.

\section{REFERENCES}

Blockey, N. J., and McAllister, T. A. (1972) Antibiotics in acute osteomyelitis in children. Journal of Bone and Joint Surgery, 54-B, 229-309. Blockey, N. J., and Watson, J. T. (1970) Acute osteomyelitis in children. Journal of Bone and Joint Surgery, 52-B, 77-87.

British Medical Journal (1972) Some problems of acute osteomyelitis. British Medical Journal, 4, 317-318.

Bulmer, J. H. (1967) Changed character of osteomyelitis. British Medical Journal, 3, 497.

Craven, J. L., Pugsley, D. J., and Blowers, R. (1970) Trimethoprim-sulphamethoxazole in acute osteomyelitis due to penicillin-resistant staphylococci in Uganda. British Medical Journal, 3, 201-203.

Green, J. H. (1967) Cloxacillin in treatment of acute osteomyelitis. British Medical Journal, 2, 414-416.

Harris, N. H. (1962) The place of surgery in acute osteomyelitis. Journal of Bone and Joint Surgery, 44-B, 219.

Jensen, K., and Lassen, H. C. A. (1969) Combined treatment with antibacterial chemotherapeutical agents in staphylococcal infections. Quarterly Journal of Medicine, 38, 91-106.

Lancet (1975) Antibiotics for osteomyelitis. Lancet, 1, 153-154.

McAllister, T. A. (1974) Treatment of osteomyelitis. British Journal of Hospital Medicine, 12, 535-545.

Merryweather, R. (1972) Acute osteomyelitis. British Medical Journal, 4, 546.

Mullick, S. (1972) Acute osteomyelitis. British Medical Journal, 4, 546.

Nade, S. (1974) Acute haematogenous osteomyelitis. Medical Journal of Australia, 2, 708-711.

Trueta, J., and Morgan, J. D. (1954) Late results in the treatment of one hundred cases of acute haematogenous osteomyelitis. British Journal of Surgery, 41, 449-457.

Weissberg, E. D., Smith, A. L., and Smith, D. H. (1974) Clinical features of neonatal osteomyelitis. Pediatrics, 53, 505-510. 